\title{
Electrical Treeing in Polymer Nanocomposites
}

\author{
Sridhar Alapati ${ }^{1}$ and M. Joy Thomas ${ }^{2}$ \\ Nanodielectrics Laboratory \\ Department of Electrical Engineering \\ Indian Institute of Science, Bangalore 560012 \\ e-mail: sridhar@ee.iisc.ernet.in, jtm@ee.iisc.ernet.in
}

\begin{abstract}
This paper presents a study of electrical treeing phenomena in polymer nanocomposites. The polymer nanocomposite studied consists of epoxy resin as the base polymer and silica as the nano filler. Treeing experiments were conducted at a constant ac voltage of $20 \mathrm{kV}, 50 \mathrm{~Hz}$ on epoxy samples without any fillers as well as epoxy silica nano composites with $1 \%$ by weight of nano silica. Time for tree inception as well as tree growth patterns were studied. The results show that addition of small amount $(1 \%$ by weight) of nano silica particles in epoxy resin can improve the treeing resistance by delaying the tree inception time as well as the time required by the tree to reach the opposite electrode. Treeing phenomena has been analyzed and interpreted by a physical model to explain the behavior in nanocomposites.
\end{abstract}

Index Terms_-Electrical Treeing, Epoxy, Nano Silica, Nanodielectrics, Nanocomposites, Polymers.

\section{INTRODUCTION}

Electrical treeing is one of the reasons for the long term degradation of polymeric insulation when stressed continuously with alternating electric field. It is a failure mechanism, where by fine erosion channels grow from a region of high electric stress (material inserts, cracks, voids and so on) with in the insulation due to partial discharge activity [1]. Once treeing occurs in the insulating material it will lead to failure when the tree channels join the opposite electrodes.

Epoxy resin is widely used as an insulation material in many electrical apparatus because of its excellent electrical and mechanical characteristics. It is usually mixed with appropriate fillers to enhance the electrical and mechanical properties. With the development of nano technology, polymer nanocomposites have drawn much attention, because they are expected to have improved properties over pure polymers or polymer micro composites by the addition of a few parts of nano fillers [2],[3].

Treeing phenomena in epoxy $\mathrm{ZnO}$ nanocomposites has been investigated by earlier researchers [4]. It was found that addition of $\mathrm{ZnO}$ particles into epoxy resin improves the resistance to electrical tree growth and also increases the time to breakdown. It has also been reported that epoxy/silica nanocomposites exhibit longer treeing life time than pure epoxy [5]. Another work reports that epoxy nanomicro particle mixed composite exhibit increased treeing life time as compared to conventional micron size particle filled as well as pure epoxy [6]. It was found that alumina nano fillers are effective in suppressing both tree initiation as well as propagation [7].

The present work focuses on the electrical treeing phenomena of the epoxy nano composite containing nano silica particles. The effect of nano filler on treeing is analyzed and interpreted by a physical model.

\section{SAMPLE PREPARATION AND PROCESSING TECHNIQUES}

\section{A. Materials}

Epoxy, one of the most widely used insulating materials in the electrical industry is used as the base polymer material in the present study. Bisphenol-A epoxy resin (CY1300) along with hardener (HY956), supplied by Huntsman was used for the investigations. One of the advantages of this particular epoxy resin is that it doesn't contain any fillers and it has a low initial viscosity. For preparing a sample using this epoxy resin and hardener, 100 parts by weight of the CY1300 resin is mixed homogenously with 25 parts by weight of the HY956 hardener. The filler used in the present study is commercially available spherical shaped silica particle of APS $15 \mathrm{~nm}$ supplied by Sigma Aldrich.

\section{B. Sample Preparation}

The specimens used for experiments were prepared by a process which included mixing, dispersion of fillers in the resin, addition of hardener, molding into a metal mould, degassing as well as cross linking and hardening by heating. It is necessary to disperse nano fillers homogeneously over the entire polymer matrix. For that, a two step procedure developed earlier [8] was used for the mixing process to prepare epoxy nanocomposites; in the first stage dispersion by mechanical mixing in a high shear mechanical mixer at a speed of $700 \mathrm{rpm}$ for different time durations but limited up to 3 minutes and in the second stage dispersion by ultrasonic agitation at a frequency of $24 \mathrm{kHz}$ for 1 hour were adopted. Then hardener is added to the particle-resin mixture and mixed thoroughly before casting it in a mould at $60^{\circ} \mathrm{C}$ for 4 hours. Before starting the preparation of the sample, the epoxy resin and the hardener were both degassed at $40^{\circ} \mathrm{C}$ for one hour to remove trapped air bubbles and moisture. Similarly, the filler particles were vacuum dried at $100{ }^{\circ} \mathrm{C}$ for 24 hours. The samples prepared are then kept in desiccators under vacuum for at least 24 hours to release residual stresses if any before carrying out the experiments. The nanocomposite samples prepared with addition of (1\% by weight) nano silica are found to be transparent enough to observe the tree growth with an optical microscope.

\section{SEM Studies}

The dispersion of fillers in the epoxy micro/nano composites were investigated by examining the sample cross-section under a JEOL field emission scanning electron microscope (FESEM). Samples of approximately $1 \mathrm{~mm} \times 1$ $\mathrm{mm} \times 1 \mathrm{~mm}$ for microscopy are prepared by cutting the epoxy composites at different locations and studied. Representative SEM micrographs are shown in figure 1 where the uniform dispersion of particles in the epoxy composites can be seen. The nano-filler dispersion shown 
confirms the effectiveness of the two-step processing method for epoxy nanocomposite sample preparation.

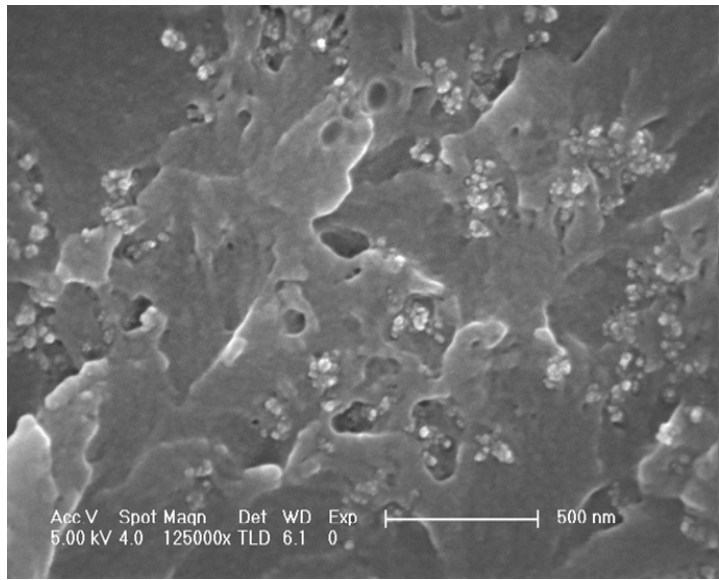

Fig. 1. SEM of epoxy silica nanocomposite (1\% by weight)

\section{Test Sample}

Test samples for treeing experiments were prepared by using a needle-plane electrode geometry by casting sharp needles into epoxy resin slabs of length $70 \mathrm{~mm}$, thickness $9 \mathrm{~mm}$ and height $12 \mathrm{~mm}$ as shown in fig. 2 . The needles used for treeing tests were $20 \mathrm{~mm}$ long, $0.5 \mathrm{~mm}$ in diameter and had a tip radius of $15 \mu \mathrm{m}$. Each needle tip was examined, measured and documented by a camera-equipped microscope before being molded into the samples. The gap distance between the needle tip and the plane electrode was adjusted to $3 \mathrm{~mm}$.

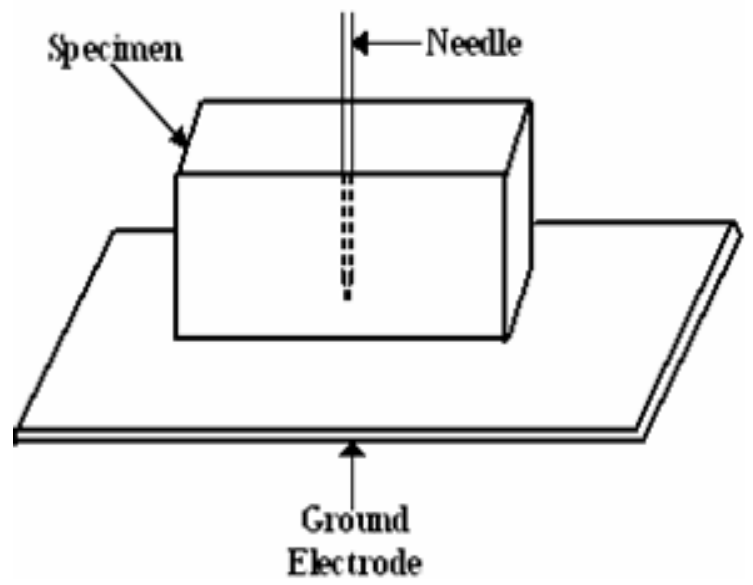

Fig. 2. Electrode arrangement for the treeing experiments

\section{EXPERIMENTAL PROCEDURE}

\section{A. Experimental Setup}

The sketch of the experimental setup for the treeing studies is shown in Fig. 3. A $30 \mathrm{kVA}, 200 \mathrm{~V} / 100 \mathrm{kV}$, transformer was used for the experiments. The experiments were conducted by applying a constant ac voltage of $20 \mathrm{kV}$, $50 \mathrm{~Hz}$ between the needle and the plane electrode and the samples were placed inside a test cell containing mineral oil to prevent surface flash over. All tests were carried out at room temperature. Figure 4 shows the photograph of the experimental setup.

\section{B. Observation of Tree Growth}

Tree inception and propagation were continuously observed through a set up developed specifically for the present work, which consists of an optical microscope, a CCD camera, and a personal computer. The microscope and the CCD camera were interfaced with a personal computer using appropriate cables. After initiation of tree in the sample, the pictures of tree growth were taken for every half an hour until tree branches reached near to the grounded electrode.

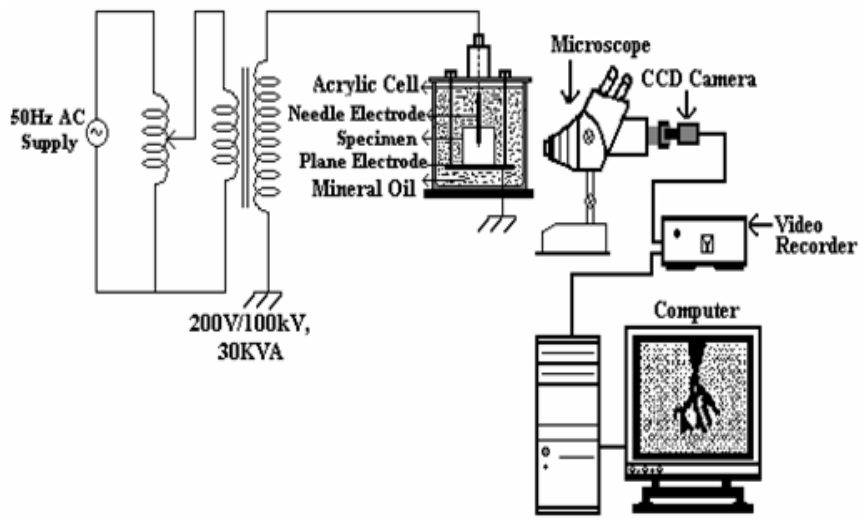

Fig. 3. Experimental setup for electrical treeing studies

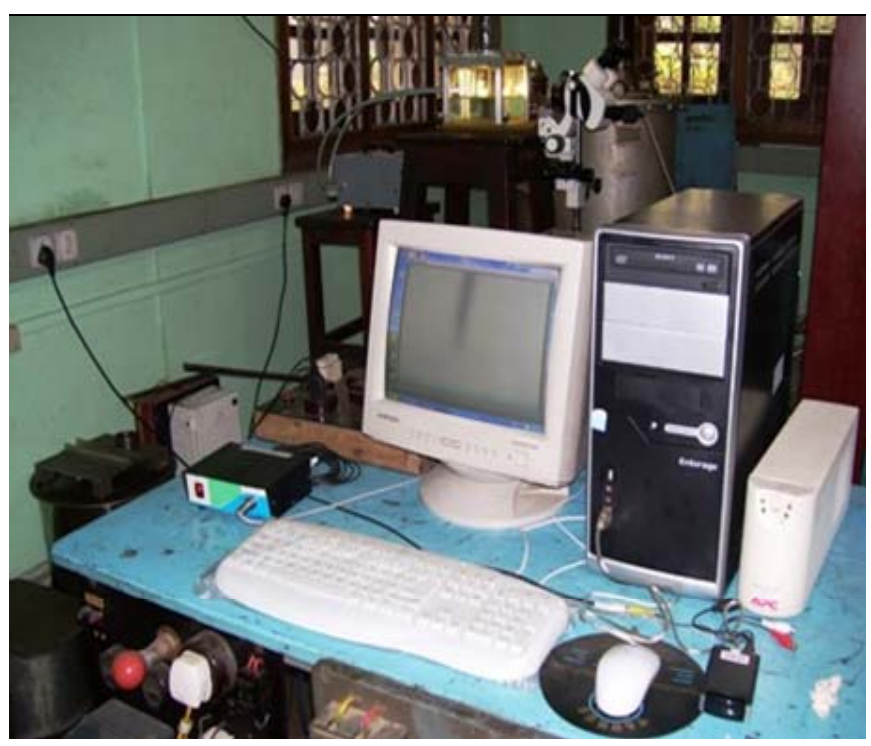

Fig. 4. Photograph of the experimental setup

\section{RESUlTS AND DISCUSSION}

Table I shows the tree initiation time for epoxy and epoxy nanocomposites. The tree initiation time for epoxy nanocomposite is more than that of pure epoxy samples and the possible reason for that is explained below.

Electrical tree growth in general can be divided into three basic periods, (1) tree incubation period, (2) tree initiation period, and (3) tree growth period. Tree incubation period is relevant only in low or medium electric fields and may not be applicable in high electric fields, when intrinsic or electron avalanche breakdown takes place. It involves different stages such as repeated process of electron injection and extraction, scission in polymer chains by injected high energy electron, oxidation of free radicals formed, and the voids formation due to repeated Maxwell stress. Addition of nano fillers increases a potential barrier 
at the interface between the high voltage electrode and the polymer composite. Also electron injection will be reduced so as to prolong tree incubation period and there by enhancing electric field strength required for tree initiation.

Table I

\begin{tabular}{|l|c|}
\hline \multicolumn{1}{|c|}{ Materials } & $\begin{array}{c}\text { Tree initiation } \\
\text { time (hours) }\end{array}$ \\
\hline Pure Epoxy & 6 \\
\hline Epoxy-Silica Nanocomposite & 10 \\
\hline
\end{tabular}

Figure 5 shows the temporal dependence of electrical tree length after the tree initiation. Length of the tree was measured in vertical direction between the tip of the electrode and the opposite plane electrode. From fig. 5, we can observe that in case of pure epoxy, tree grew as time passed by leading to breakdown after 1 hour of tree initiation. In the case of epoxy nanocomposite, tree length was less than $500 \mu \mathrm{m}$ at 1 hour after tree initiation. The tree pattern also changed from branch type to bush type after 1 hour of tree initiation.

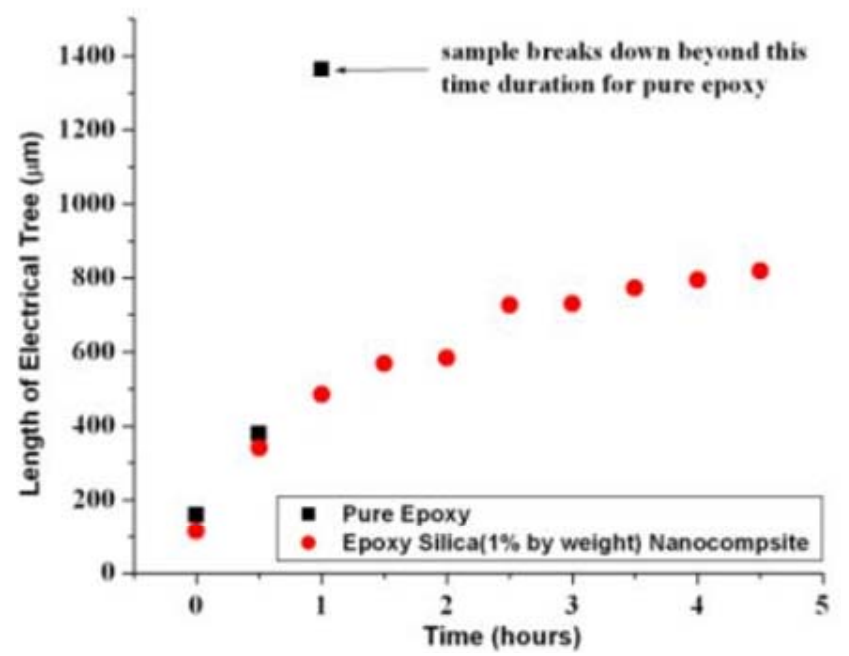

Fig. 5. Propagation of electrical tree after tree initiation

Figures 6 and 7 show photos of tree growth patterns for pure epoxy as well as epoxy silica nanocomposites after application of the voltage. From fig. 6 and fig. 7, we can observe that tree grows rapidly in pure epoxy and it will reach opposite electrode after one hour of tree initiation but in case of epoxy nanocomposite tree growth is fast initially and becomes slower after 1 hour of tree initiation and it does not reach the opposite electrode even after 4 hours of tree initiation. From fig. 6 and fig. 7 we can also observe that the tree growth patterns are different for both the pure epoxy as well as the epoxy nanocomposites. In pure epoxy, the tree pattern looks like a branch type tree with a single main branch channel where as in the case of epoxy nanocomposites, initially it forms a branch type of tree and later on become dense by growing in lateral directions to form a bush type of tree with time. The reason for this type of pattern is explained by a physical model.

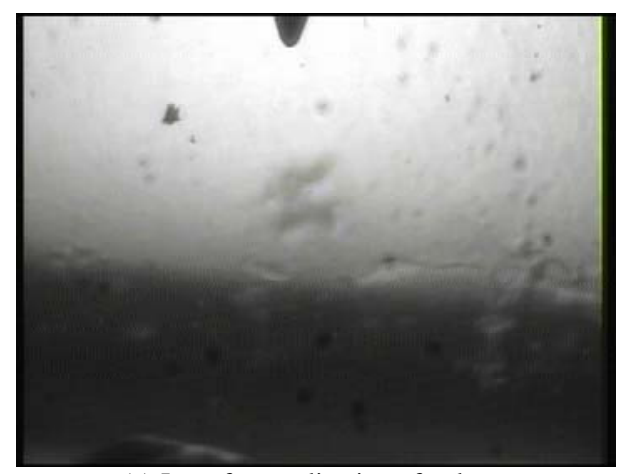

(a) Just after application of voltage

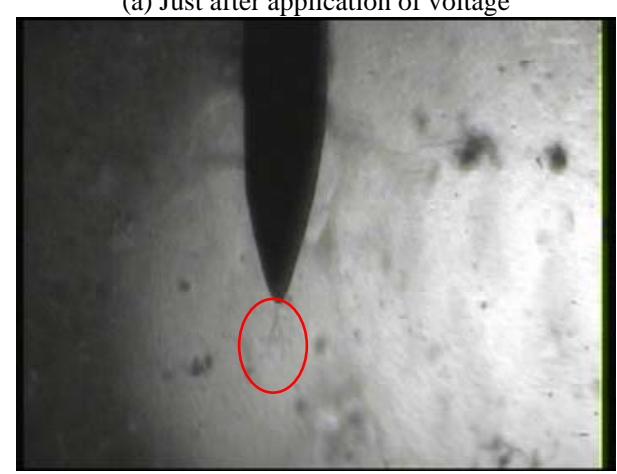

(b) Just after tree initiation

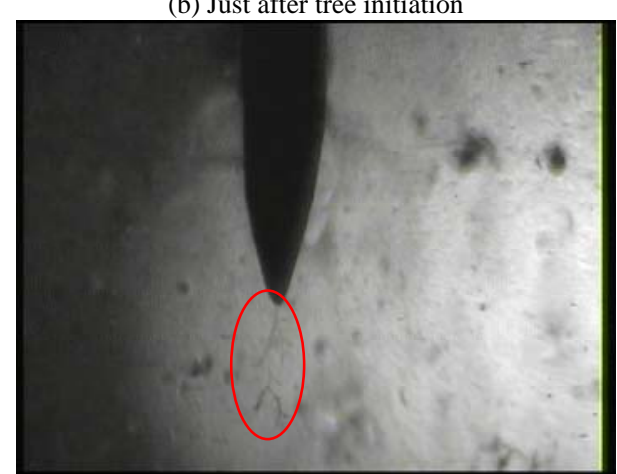

(c) After $1 / 2 \mathrm{~h}$ of tree initiation

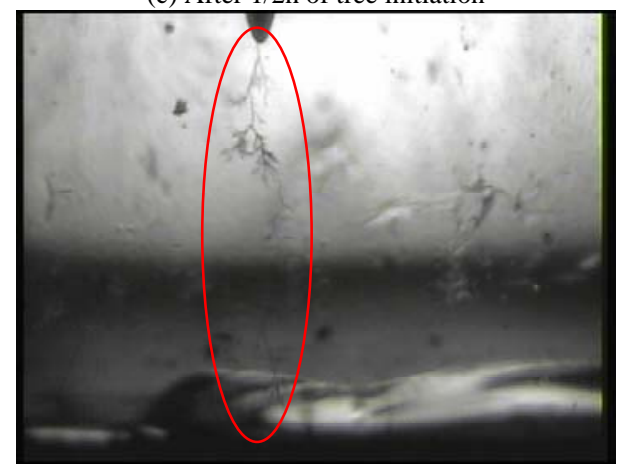

(d) After 1h of tree initiation (just before breakdown)

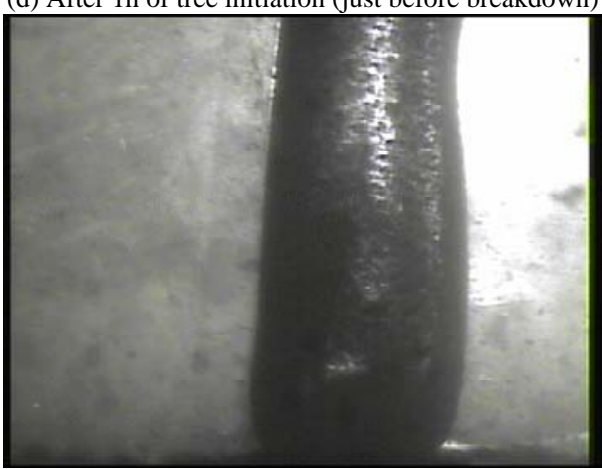

(e) Tree channel after breakdown

Fig. 6. Electrical tree growth pattern in pure epoxy. Tree growth can be seen inside the circle 


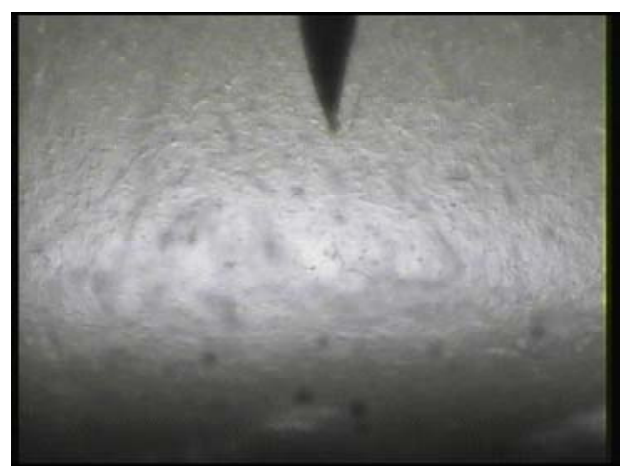

(a) Just after application of voltage
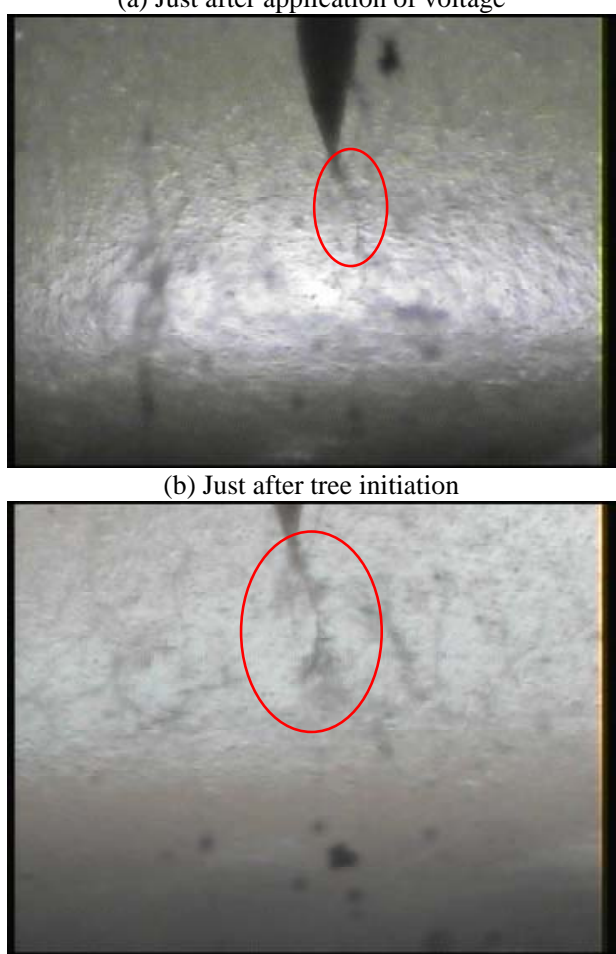

(c) After $1 / 2 \mathrm{~h}$ of tree initiation

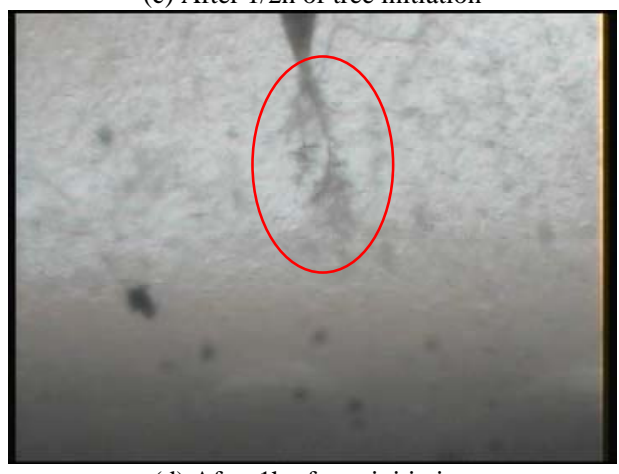

(d) After $1 \mathrm{~h}$ of tree initiation

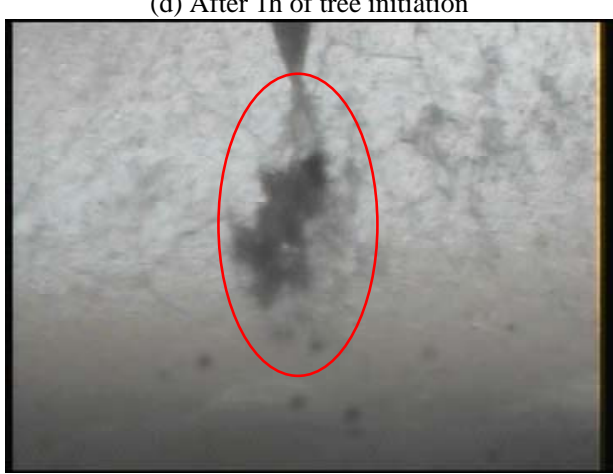

(e) After $4 \mathrm{~h}$ of tree initiation

Fig. 7. Electrical tree growth pattern in epoxy-silica nanocomposite. Tree growth can be seen inside the
Figure 8 shows a possible physical model for tree growth mechanism in filled epoxy. After initiation of tree, it starts to grow through the resin and during this process if it collides with the filler it will dwell at the filler surface and try to grow through the filler-epoxy interface. The fillers would create an obstruction to the propagation, and prevent tree from growing straight as fillers are more resistant to partial discharge as compared to the resin. After propagating along the interface, the tree continuously propagates to another filler through the resin as shown in fig. 8. Therefore, tree pattern tends to be more zigzag and it becomes denser. By the addition of nano filler, the number density of the filler for a given volume will increase. It leads to increase in frequency of tree channels colliding with the particles, and hence the tree pattern is more bushy.

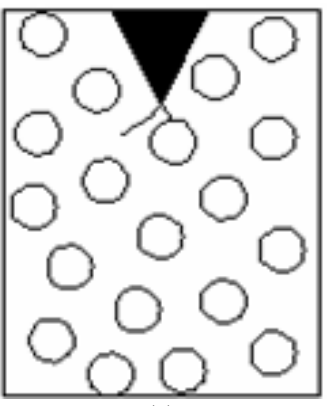

(a)

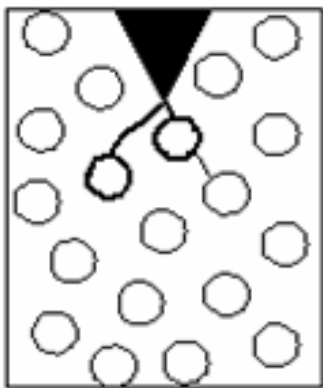

(c)

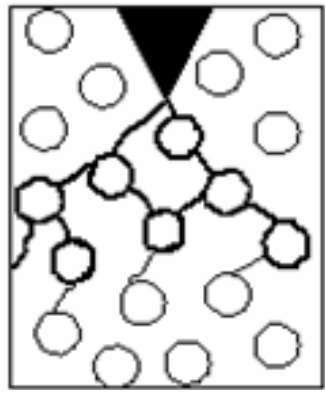

(e)

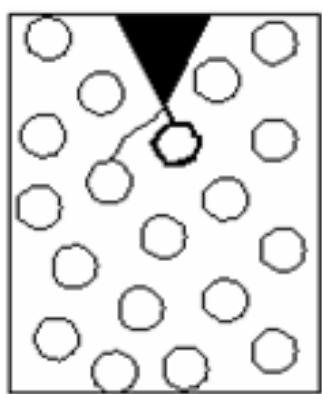

(b)

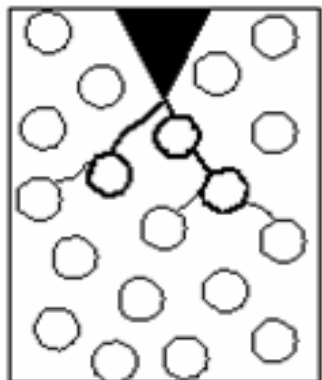

(d)

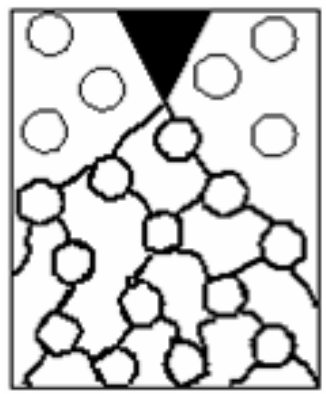

(f)
Fig. 8. Physical model for tree growth mechanism in filled epoxy

\section{V.CONCLUSION}

Treeing phenomena in pure epoxy and epoxy silica nanocomposite were experimentally investigated. It was observed that addition of small amount ( $1 \%$ by weight) of nano filler will improve the tree growth resistance of the polymer. The effect of fillers on tree growth was explained by using a physical model. The effect of different filler concentrations, filler materials, and filler size on treeing has to be examined in order to understand the treeing phenomena better in polymer composites. 


\section{REFERENCES}

[1] L. A.Dissado and J. C. Fothergill, Electrical Degradation and Breakdown in Polymers, G.C. Stevens, Ed., Peter Peregrinus, London (1992).

[2] T. Tanaka, G. C. Montanari, and R. Mulhapt, "Polymer Nanocomposites as Dielectrics and Electrical Insulation -Perspective for Processing Technologies, Material Characterization and Future Applications”, IEEE Transactions on Dielectrics and Electrical Insulation, Vol.11, No.5, pp 763-784, October 2004.

[3] T. Tanaka, "Dielectric nanocomposites with Insulating Properties", IEEE Transactions on Dielectrics and Electrical Insulation, Vol.12, No.5, pp 914-928, October 2005.

[4] H. Z. Ding and B. R. Varlow, "Effect of Nano Fillers on Electrical Treeing in Epoxy Resin Subjected to AC Voltage”, IEEE Conference on Electrical Insulation and Dielectric Phenomena, Boulder, Colorado, USA, October 17-20, 2004, pp.332-335.

[5] T. Imai, F. Sawa, T. Ozaki, T. Shimizu, S. Kuge, M. Kozako, and T. Tanaka, "Effect of Epoxy/Filler Interface on Properties of Nano or Microcomposites", IEEJ Transactions on Fundamentals of Materials, Vol.126, No.2, pp.84-91, 2006.

[6] T. Imai, F. Sawa, T. Nakano, T. Ozaki, T. Shimizu, M. Kozako, and T. Tanaka, "Effect of Nano and Micro-filler Mixture on Electrical Insulation Properties of Epoxy based Composites", IEEE Transactions on Dielectrics and Electrical Insulation, Vol.13, No.1, pp.319-323, February 2006.

[7] T. Tanaka, A. Matsunawa, Y. Okhi, M. Kozako, M. Kohtoh, and S.Okabe, "Treing Phenomena in Epoxy/Alumina Nanocomposites and Interpretation by a Multicore Model”, IEEJ Transactions on Fundamentals of Materials, Vol.126, No.11, pp.1128-1134, 2006.

[8] Santanu Singha and M. Joy Thomas "Dielectric Properties of Epoxy Nanocomposites", IEEE Transactions on Dielectrics and Electrical Insulation, Vol.15, No.1, pp.12-23, February 2008.

Sridhar Alapati was born in Andhrapradesh, India in 1982. He received the B.E. degree in Electrical Engineering from QIS College of Engineering and Technology, Ongole, India in 2004 and the M.Tech degree in Power Apparatus and Systems from the Institute of Technology, Nirma University, Ahmedabad, India in 2006. Since August 2006, he is a fulltime graduate student at the Department of Electrical Engineering, Indian Institute of Science, Bangalore, India working towards his $\mathrm{PhD}$ degree in dielectrics and electrical insulation technology. His areas of interest are Nano dielectrics, High voltage rotating machine as well as cable insulation and Design of electrical insulation systems for HV equipments.

M. Joy Thomas was born in Kerala, India in 1961. He received the B.Tech. degree in Electrical Engineering from Institute of Technology, BHU, Varanasi, India in 1983, the M.Sc. (Engg.) and Ph.D. degrees from Indian Institute of Science, Bangalore, India. Presently, he is working as Assistant Professor at the High Voltage Laboratory, Department of Electrical Engineering, Indian Institute of Science, Bangalore, India. He is a member of IEEE, CIGRE and ASEE. His areas of interest are EHV/UHV power transmission engineering, High voltage engineering, Dielectrics and electrical insulation, Asset management and condition monitoring of high voltage power apparatus, Pulsed power engineering, Plasma science and technology, EMC and Engineering pedagogy. 